$\begin{aligned} & \text { Edutec. Revista Electrónica de } \\ & \text { Tecnología Educativa } \\ & \text { Núm. 16./Abril } 03\end{aligned}$

\title{
AGENTES INTELIGENTES EN EDUCACIÓN
}

Gonzalo Villarreal Farah

Centro Comenius Universidad de Santiago de Chile gvillarr@comenius.usach.cl

\section{RESUMEN}

Diferentes sistemas computacionales tienen como finalidad apoyar el proceso de enseñanza y en particular de aprendizaje de los estudiantes. Sin embargo, son pocas las aplicaciones que pueden demostrar que apoyan en el logro de los contenidos y/o habilidades propuestos. En el área de la inteligencia artificial, por mucho tiempo, los sistemas tutores inteligentes, fueron desarrollados teniendo algún nivel de resultado. Sin embargo, en la última década aparecen un nuevo tipo de sistemas, basados en agentes inteligentes, que han demostrado su efectividad en procesos de formación y apoyo a la labor tanto de los profesores como de los alumnos. Estos sistemas, se comportan no sólo como un tutor inteligente, si no como un miembro más de un grupo de estudiantes. A raíz del desarrollo que ha experimentado la tecnología en los últimos años y de los resultados de las investigaciones sobre estos agentes inteligentes, se ha observado una creciente atención por estos sistemas. En este artículo, se presentan algunas líneas de investigación del área, como lo son las interfaces basadas en agentes inteligentes, agentes pedagógicos, "compañeros de aprendizaje" y los hipertextos adaptativos.

\section{INTRODUCCIÓN}

En muchos países, la educación ha sido y esta siendo fuertemente influenciado por la inserción de la tecnología. Esto se puede observar, entre otros, en: la optimización de recursos; la mejora de los procesos de enseñanza aprendizaje; la una educación más equitativa; generar una formación continua; y mejorar la "sintonía" entre la escuela y la sociedad (Bruner, 2000).

De esta manera, la tecnología esta influenciando al menos en dos aspectos al mundo educacional. Uno relacionado con los intereses pedagógicos, administrativos y de gestión escolar y la segunda con los cambios en las habilidades y competencias requeridas, para lograr una inserción de las personas en la sociedad actual.

Lo anterior, presenta un escenario de crecientes necesidades de apoyo a la formación de los estudiantes, donde, por una parte, con menores o mayores avances, hay tecnología ya instalada (computadores, Internet, software de propósito general y algunas aplicaciones educativas) y por otra los procesos de alfabetizado de los profesores.

El software educativo, juega un papel importante como apoyo al docente y al estudiante en la formación de este último. Si bien existen importantes y numerosos desarrollos de sistemas, los resultados no han sido lo esperado. En su mayoría corresponden a sistemas de práctica y ejercitación y su principal característica es entregar al alumno la posibilidad de ejercitarse en una determinada tarea una vez obtenidos los conocimientos necesarios para el dominio de la misma (Gros, 1997).

Los sistemas de ejercitación y práctica, no tienen una característica formativa. Sin embargo, existe una línea de sistemas desarrollados para apoyar la formación de los alumnos denominados tutores inteligentes. Por diferentes motivos, estos no han logrado los resultados esperados, dándole paso a sistemas que se basan en agentes inteligentes, los cuales son capaces de: comunicarse con el usuario en un lenguaje natural; simular el comportamiento humano; adaptarse a las necesidades del alumnos; entre otros, lo que 
le ha permitido a estos sistemas apoyar la formación de los estudiantes, con la utilización de nuevos modelos y herramientas.

Este artículo trata sobre los agentes inteligentes en el área de educación, en particular, se presentan algunos desarrollos y líneas de trabajo, como lo son las interfaces basadas en agentes inteligentes, agentes pedagógicos, sistemas denominados "compañeros de aprendizaje" y los hipertextos adaptativos.

\section{LA INTELIGENCIA ARTIFICIAL}

Inteligencia Artificial (IA), es el área de investigación de la cual nacen diferentes desarrollos entre los que se encuentran los agentes inteligentes.

El concepto de IA, se refiere al diseño de sistemas informáticos inteligentes, es decir, que poseen las características comúnmente asociadas a la inteligencia humana: comprensión del lenguaje natural, capacidad de solución de problemas y de aprendizaje, razonamiento lógico, entre otros.

Existen innumerables definiciones de IA, sin embargo se usará la siguiente: "La automatización de actividades que asociamos con el pensamiento humano, actividades como la toma de decisiones, la resolución de problemas, el aprendizaje, etc." (Bellman, 1978).

La IA aparece con la finalidad de: a) duplicar las facultades del comportamiento que atribuimos al ser humano, entendido como ser con capacidad de pensar; b) resolver problemas reales, actuando como un conjunto de ideas acerca de cómo representar y utilizar el conocimiento y de cómo desarrollar sistemas informáticos; y c) buscar la explicación de diversas clases de inteligencia, a través de la representación del conocimiento y de la aplicación que se da a éste en los sistemas informáticos desarrollados.

Algunas áreas de la IA son: Robótica; Procesamiento de lenguaje natural; Reconocimiento de patrones; Sistemas Expertos; Agentes inteligentes; Tutores inteligentes; Demostración de teoremas; Redes neuronales; Manipulación inteligente de base de datos; Web inteligentes; Minería de datos; Programación automática; Visión computarizada; entre otros.

En particular, se tratará el tema de agentes inteligentes relacionados con el ámbito educativo.

\section{Agentes inteligentes}

Una estrategia de acercamiento de las tecnologías a los usuarios, se encuentra en los desarrollos que se han realizado de agentes inteligentes, del área de la inteligencia artificial. Son conocidas las metáforas referenciadas por Negroponte respecto a la capacidad de relación entre las personas y los computadores, en la capacidad de estos de manejar la información y fíltrala según los intereses y necesidades de cada usuario (Negroponte, 1995).

Existen diferentes definiciones o significados de agentes inteligentes, dependiendo principalmente del dominio del conocimiento en el cual se refiere. Nos referiremos a agentes inteligentes como fragmentos de software con características humanas que facilitan el aprendizaje. Las características pueden expresarse desplegando texto, gráfico, iconos, voz, animación, multimedio o realidad virtual (Choua, Chanb y Linc, 2002).

Dos aplicaciones típicas de agentes inteligentes son los sistemas tutores inteligentes (systems intelligent tutors - ITS) y los "compañero de aprendizaje", (learning companion system - LCS). Los ITSs simulan a un tutor autoritario que posee una estrategia de enseñanza uno a uno, que es un experto en un dominio del conocimiento y actúa como un guía, tutor o un entrenador. Este tutor, puede adaptarse según las necesidades del estudiante. Los LCSs, son agentes pedagógicos no autoritarios, no es experto en un dominio e incluso puede cometer errores. Se adoptan actividades de aprendizaje colaborativas o competitivas, como alternativas de un tutor uno a uno. En alguno de sus roles, puede actuar igual como un tutor, como un capaz estudiante/profesor (estudiante que enseña a otros estudiantes), colaborador, competidor, alborotador, crítico o clon (Choua et al., 2002).

Estos sistemas pueden ayudar a la labor del docente o del alumno, sin involucrarse directamente en la actividad instructiva. Un profesor auxiliar, puede proporcionarle al profesor, la carpeta de aprendizaje de los estudiantes, incluso la actuación de aprendizaje, las equivocaciones, nivel de esfuerzo y motivaciones, entre otros aspectos. Este archivador puede ayudar al profesor a entender al estudiante y responder apropiadamente a cada uno. El estudiante auxiliar, por ejemplo, puede ayudar al estudiante a coleccionar información para realizar la actividad de aprendizaje.

Los agentes inteligentes, proporcionan a los estudiantes recursos que pueden clasificarse en tres categorías: contenidos (libros, bibliotecas, museos, bases de datos, etc.); soporte computacional (calculadora, software, etc.); y comunidad (compañeros de estudio; profesores; padres; voluntarios; etc.). De esta manera el estudiante dispone de múltiples medios y de un contexto, por lo que el agente es 
presentado en un medio ambiente de aprendizaje social (Choua et al., 2002). Un agente educativo no sólo puede imponer directamente sus perspectivas en el estudiante, sino que también estimula el aprendizaje y colabora entre los estudiantes humanos.

A continuación se presenta una tabla que permite observar la similitud y diferencia entre la arquitectura de los sistemas ITSs y los sistemas LCSs (cuya arquitectura es conocida con el nombre de GCM).

Tabla 1. Comparación de las componentes típicas de un ITS y la arquitectura de un GCM (Choua et al., 2002, pág. 263).

\begin{tabular}{|l|l|l|}
\hline \multicolumn{1}{|c|}{ Componente } & \multicolumn{1}{|c|}{ Similitud } & \multicolumn{1}{c|}{ Diferencia } \\
\hline Modelo del estudiante (ITS) & $\begin{array}{l}\text { Ayuda a comprender el estado del } \\
\text { estudiante }\end{array}$ & $\begin{array}{l}\text { Desarrollado desde el punto de } \\
\text { vista de un tutorDesarrollado desde } \\
\text { el punto de vista de un tutor o un } \\
\text { compañero }\end{array}$ \\
\hline El módulo de enseñanza (ITS) & $\begin{array}{l}\text { Determina la conducta pedagógica } \\
\text { del agente }\end{array}$ & $\begin{array}{l}\text { El agente actúa como un tutorEl } \\
\text { agente puede actuar como un } \\
\text { competidor, igual que un tutor, ser } \\
\text { crítico, un alborotador o colaborador }\end{array}$ \\
El módulo pedagógico(GCM) & $\begin{array}{l}\text { El compañero puede tener la } \\
\text { competencia de un excelente } \\
\text { estudiantes, un estudiante medio o } \\
\text { un novicio }\end{array}$ \\
\hline $\begin{array}{l}\text { Módulo de dominio del experto } \\
\text { (ITS) } \\
\text { Módulo de dominio(GCM) }\end{array}$ & dominio del agente & $\begin{array}{l}\text { Provee las características del } \\
\text { compañero }\end{array}$ \\
\hline $\begin{array}{l}\text { El modelo del compañero de } \\
\text { aprendizaje (GCM) }\end{array}$ & & \\
\hline
\end{tabular}

De esta manera, los desarrollos de inteligencia artificial, en particular los referidos a los agentes inteligentes, se han investigado viendo como una alternativa real de apoyo a los procesos de enseñanza aprendizaje de los estudiantes

\section{Líneas de investigación de los agentes inteligentes}

A continuación, y a modo de ejemplo, se presentan algunas descripciones de áreas donde actúan los agentes inteligentes en educación. Con esto se espera entregar una visión del potencial de estos sistemas y presentar en forma resumida el estado actual del área. Así, se presentarán los siguientes temas:

- Interfaces que utilizan agentes inteligentes

- Agentes pedagógicos, en particular los conocidos como "Compañero de
aprendizaje"

- Hipertextos adaptativos

En forma sucesiva, se están desarrollando diferentes productos tecnológicos que incluyen agentes inteligentes, los cuales van desde poder entregar información más relacionada con los estudiantes, manejo de datos del usuario ya sea para reconocerlo y/o para tomar decisiones futuras, apoyarlo en el desarrollo de la sesión de trabajo e incluso ayudarlo como cuando un compañero enseña a otro, etc.

Una línea interesante de presentar, es el trabajo de Justine Cassell del Instituto Tecnológico de Massachussets, quien ha desarrollado sistemas con agentes inteligentes, en particular de interfaces, que pueden tomar la información y representarla con discurso, con gesto, con expresión facial, con postura, etc. Estos sistemas son capaces de entender gestos de manos, faciales, detectar la presencia o ausencia del usuario, si lo conoce o no, entre otros elementos.

Estos sistemas permiten relacionarse de mejor manera con las personas. Por ejemplo, para un estudiante que ingresa a una página web y ve una interfaz que presenta un rostro humano, con el que se puede comunicar por medio del lenguaje verbal o escrito (en lenguaje natural), que es capaz de entenderlo y seguir la conversación y orientarlo en sus necesidades, entre otros elementos, al usuario humano que es principiante o inexperto, le será más gratificante, más natural, menos frustrante, más orientador y seguramente se sentirá más acogido. Para Cassell, es una de las maneras de lograr confianza con el usuario, haciendo que la máquina se comporte como los seres humanos (Ryokai, Vaucelle y Cassell, 2002a).

Los desarrollos de Cassell y su extensa investigación en el área del comportamiento 
conversacional humano, han permitido generar avances en la automatización de manera que los agentes virtuales posean una voz, aplicando reglas al discurso y a las diferentes formas de expresiones (Ryokai et al., 2002a; Ryokai et al., 2002b).

Otros avances en esta línea, son sistemas que animan a niños a desarrollar y contar sus historias, desarrollando la capacidad de representar pensamientos simbólicamente y de compartirlos con otros niños en su propio lenguaje. Uno de estos desarrollos, el "Storytelling" es un sistema que apoya el trabajo en forma colaborativa entre niños, proporcionando un ambiente para que estos aprendan las habilidades más importantes del lenguaje.

A partir de esta experiencia, el grupo de trabajo de Cassell, generaron una investigación donde diseñaron SAM, el cual es un agente conversacional que puede contar historias en forma colaborativa con los niños. Este sistema, pensado para ser un niño más del grupo curso de preescolares, incentiva a contar historias de una manera desarrollada mentalmente de forma más avanzada, modelando las habilidades narrativas importantes durante el proceso de aprendizaje (Ryokai et al., 2002b).

Los resultados de las investigaciones de Cassell, demostraron que los niños que jugaron con el "niño virtual" desarrollaron historias que se acercan más a historias lingüísticas avanzadas, además estos niños usaron en sus historias, un número mayor de expresiones temporales y espaciales. También, los niños escucharon las historias de SAM cuidadosamente, junto con intervenir y sugerir cambios a estas. Sin embargo, las ventajas potenciales de contar con estos sistemas, aun están en discusión (Ryokai et al., 2002b).

Otra línea de desarrollo son los agentes pedagógicos animados que nacen de los sistemas basados en conocimientos y los sistemas de interfaces inteligentes, como los mencionados anteriormente. En estos sistemas, los estudiantes pueden aprender y pueden practicar determinadas habilidades en un mundo virtual y el sistema puede actuar por medio de un dialogo simulando a un tutor o enseñar como si fuera uno de sus compañeros (Johnson, Rickel y Lester, 2000).

Los sistemas que apoyan a los estudiantes son conocidos con nombres tales como "compañero de aprendizaje", (learning companion system -LCS), "co-aprendiz", "simulación del estudiante" y "estudiante artificial".

En especial, estos sistemas que apoyan a estudiantes desde la perspectiva de un compañero, pueden colaborar o competir con el estudiante humano. Se pueden definir tres estrategias de usos de estos sistemas: cuando el agente inteligente, trabaja en forma independiente con una perspectiva de competición; cuando el agente inteligente colabora por medio de sugerencias; y cuando, por medio de una colaboración activa, con responsabilidad compartida, participa y apoya al estudiante humano. Incluso, se ha propuesto que estos sistemas permiten acercarse a la idea, que el estudiante humano "aprenda a aprender", en la medida que estos últimos enseñan al sistema (a su compañero de aprendizaje). En este punto, se trata que el estudiante proporcione conocimientos y ejemplos al sistema, de esta manera podrá observar como este resuelve los problemas, y luego, el estudiante explica si la solución es correcta o no y por qué (Choua et al., 2002).

Se han desarrollado múltiples LCS, con diversas finalidades. Desarrollos de diferentes agentes, para motivar a distintos grupos, agentes que enseñan en procesos de aprendizajes sociales, agentes que son alborotadores e incluso proporcionan opiniones erróneas. Estos últimos, pretenden entregar un elemento disonante entre lo que el estudiante sabe y lo que el agente le sugiere, de manera que obligan al estudiante a buscar nueva información para reducir la disonancia (Choua et al., 2002).

Se ha observado, la importancia de la comunicación no verbal en los procesos de enseñanza. De esta manera, estos agentes pedagógicos, aprovechan por medio de la mirada y gestos, llamar la atención del estudiante. Mediante movimientos de cabeza y expresiones faciales, puede entregar señales y feedback claros al estudiante sin interrumpir su pensamiento. Esto los obliga a ser naturales, creíbles y parecer un ser humano. En definitiva, estos sistemas permiten aumentar los canales de comunicación (bandwidth) entre el computador y los estudiantes, junto con aumentar la habilidad del sistema de comprometerse y motivar a los estudiantes (Johnson et al., 2000).

Al actuar estos agentes en medios educacionales, para apoyar las interacciones de aprendizajes, la dificultad para estos sistemas no es sólo la de realizar la tarea, sino que se le exige un conocimiento y entendimiento profundo de cómo y por que actuar frente a cada intervención con o del usuario (Johnson et al., 2000).

Los sistemas con agentes inteligentes poseen diferentes características que son de gran 
- Estos sistemas permiten actuar y dialogar con los agentes, de manera que en el momento de realizarse acciones, estas pueden ser vistas de diferentes ángulos.

- Los estudiantes pueden realizar preguntas en cualquier momento.

- El agente esta en todo momento "observando" el actuar del estudiante.

- El agente puede reconstruir y redefinir su actuar en cada momento a partir del actuar del estudiante.

- El agente puede adaptar su actuar a situaciones inesperadas.

- El estudiante puede tomar el control en cualquier momento.

- En caso de errores el agente ayuda a que el estudiante aprenda de ellos.

- En cualquier momento, el estudiante puede consultar en que parte del proceso se encuentra y las tareas faltantes.

Existen otras variadas ventajas de estos agentes tales como el manejo de emociones, apoyo efectivo del trabajo colaborativo, interacciones pedagógicas adaptables, etc. (Ryokai et al., 2002a; Ryokai et al., 2002b).

\section{Hipertextos Adaptativos}

Como una estrategia particular de utilización de los agentes inteligentes en sistemas educacionales, está el desarrollo de los hipertextos adaptativos. Si bien existen hipertextos que no poseen agentes inteligentes, estos presentan una serie de dificultades que pueden ser resueltas por la utilización de estos agentes.

Un Hipertexto es un documento hipermedia, como un sitio Web educativo o un CD-ROM que contienen un cuerpo coherente de materia organizada y desarrollado con propósitos educativos (Murray, Condit, Piemonte, Shen \& Khan, 2000).

Un elemento importante de los hipertextos adaptativos, es la posibilidad de individualizar el acceso a la información de manera que esta se acomode y responda a la diversidad y necesidades de los usuarios posibles, junto con ser capaz de modelar al alumno y ser capaz de actualizar dicha información. Si en los sistemas tradicionales de instrucción, el alumno accede a la información en forma lineal, aquí el alumno decide la manera en que accede. Así, el estudiante no tiene a su disposición sólo la estructura del diseñador o del instructor, sino que puede elegir la manera de acceder, interactuar o interrelacionarse con la información, dependiendo de sus experiencias previas y habilidades. De esta manera, la información se ajusta a las necesidades del alumno, en lugar que este se ajuste a la información (Pérez, Gutiérrez, López, González y Vadillo, 2001).

Los problemas típicos de estos sistemas, para los usuarios son: la primera, producida por la desorientación del usuario, referida a no saber en que espacio del hipertexto se encuentra, desde donde viene, para donde va, etc. La segunda, se refiere a una carga excesiva cognoscitiva, la que se produce cuando el estudiante se angustia frente a las diferentes posibilidades de navegación del hipertexto y de herramientas disponibles. La tercera, se refiere al flujo discontinuo, cuando los saltos en la navegación pueden producir interrupciones en el flujo narrativo y conceptual del material, pudiendo confundir al lector (Murray et al., 2000).

Al menos dos problemas de los propuestos por Lewis, en el uso de cursos de instrucción desarrollados en Web, refiriéndose a cursos basados en hipertextos, son interesantes de mencionar: a) el acceso a los materiales del curso es lento; y b) el curso no se adapta automáticamente a cada estudiante (Johnson \& Shaw, 1997).

Los desarrollos de hipertextos más inteligentes, han permitido que se adapten y/o proporcionen guías explícitas a los estudiantes. Además los documentos hipermedia se adaptan de manera rápida, personalizándose al estilo y secuenciación según las necesidades del usuario. Otros proporcionan guías o feedback respecto a los progresos y trayectorias de los estudiantes (Murray et al., 2000). 
Existe un creciente aumento de formación, tradicional, a distancia, educación continua, entre otros, la cual permite ver a estos sistemas basados en agentes inteligentes como sistemas de apoyo a dichos procesos formativos, permitiendo desarrollar mejores seguimientos, feedback, interacción con cada usuario, manejo de una visión del grupo y de cada estudiante, manejo de grupos, incentivos, adaptación a las necesidades, etc.

Existen diferentes experiencias de investigación de agentes inteligentes, que están siendo apoyo en la relación entre los usuarios humanos y los sistemas computacionales.

Las investigaciones señalan que algunas de las dificultades presentadas en los desarrollos de hipertextos que no usan agentes inteligentes, son factibles de solucionarse con la inclusión de estos agentes.

Se ha observado que las herramientas basadas en hipertexto, son de utilidad para el trabajo de tipo constructivista, colaborativo y cooperativo de los estudiantes (Pérez et al., 2001).

Las investigaciones han presentado que los sistemas basados en agentes inteligentes, requieren de menor tiempo de desarrollo que otros sistemas inteligentes, como los tutores inteligentes.

Si bien, se esta observando algunos resultados de las investigaciones que usan esta tecnología, estos resultados auguran la posibilidad de que estos sistemas puedan ser utilizados efectivamente como agentes pedagógicos, generando impactos positivos en la educación de los estudiantes.

Son notables los resultados de los sistemas de interfaces con agentes inteligentes, donde muchos de los cuales interactúan con niños preescolares, con buena aceptación por parte de estos, como otro niño del grupo, y logrando aprendizajes importantes en las áreas de investigación. Estos agentes han permitido que los sistemas se vean más humanos, que actúen más como estos y sea factible aumentar los canales de comunicación entre hombre y máquina, lo que permitirá simular, en estos sistemas, muchas de las estrategias que se utilizan en los procesos educativos entre profesor y alumno.

Para el desarrollo de muchos de estos sistemas, entre otros, se requerirá teóricos de la comunicación, lingüistas, especialistas en gráficos y animación (Johnson et al., 2000).

Sin embargo, cabe señalar que si bien estos sistemas están logrando resultados interesantes, estos deben ser insertados bajo una filosofía y un modelo pedagógico sólido. Lo que esta sobre todo tratamiento pedagógico de un tema, es contar con un buen modelo pedagógico (probado y validado) (Nagel, 2002).

\section{REFERENCIAS}

Bellman, R.E. (1978) An introduction to Artificial Intelligence: Can Computers Think? San Francisco: Boyd \& Fraser Publishing Company.

Bruner, J.J., (2000). Educación: Escenarios de Futuro. Nuevas Tecnologías y Sociedad de la Información. PREAL: Promoción de la Reforma Educacional en América Latina y el Caribe. Disponible en http://www.preal.cl/brunner16.pdf

Cassany, D. (2002). La alfabetización digital. Ediciones de la Universidad Pompeu Fabra, Barcelona.

Castells, M. (2002). Lección inaugural del programa de doctorado sobre la sociedad de la información y del conocimiento, Documento presentado en Inauguración doctorado sociedad de la Información, Barcelona: Editorial UOC. Disponible en http://www.uoc.edu/web/esp/articles/castells/menu10.html

Choua, C., Chanb T. \& Linc C. (2002). Redefining the learning companion: the past, present, future of educational agents, Computer \& Education, 40(3), 255-269.

Gros, B. (1997) Diseños y programas educativos Pautas pedagógicas para la elaboración de software (enero 1997). Barcelona: Ariel Educación.

Johnson W.L. \& Shaw E. (1997). Using Agents to Overcome Deficiencies in Web-Based Courseware. Documento presentado en Proceedings of the workshop "Intelligent Educational Systems on the World Wide Web", 8th World Conference of the AIED Society, Kobe, Japan, 18-22. 
Johnson W.L, Rickel J.W. \& Lester J.C. (2000). Animated Pedagogical Agents: Face-to-Face Interaction in Interactive Learning Environments, International Journal of Artificial Intelligence in Education, 11(2000), 47-78.

Murray T., Condit C., Piemonte J., Shen T. \& Khan S. (2000). Evaluating the Need for Intelligence in an Adaptive Hypermedia System, Documento presentado en ITS Montréal 2000.

Naciones Unidas (2001) Informe Anual para el Desarrollo Humano, Poner el adelanto tecnológico al servicio del desarrollo humano. Nueva York: Ediciones Mundi-Prensa. Disponible en http://www.undp.org/hdr2001/spanish/

Naciones Unidas (1998). Informe Anual para el Desarrollo Humano, Consumo para el desarrollo. Nueva York: Ediciones Mundi-Prensa.

Nagel, G.K. (2002). Building Cultural Understanding and Communication: A Model in Seven Situations. Disponible en http://www.readingonline.org/newliteracies/lit index.asp? HREF=/newliteracies/nagel

Negroponte, N (1995) El Mundo digital. Barcelona: Ediciones B.

Pérez T.A., Gutiérrez J., López R., González A. \& Vadillo J. A. (2001). Hipermedia, adaptación, constructivismo e instructivismo. Revista Iberoamericana de Inteligencia Artificial, 3(2), 29-38.

Poole, B. (1999) Tecnología Educativa. Educación para la sociedad de la comunicación y del conocimiento, Madrid: Mc Graw Hill.

Ryokai, K., Vaucelle, C., \& Cassell, J. (2002a). Literacy Learning by Storytelling with a Virtual Peer. Documento presentado en In Proceedings of Computer Support for Collaborative Learning 2002. Disponible en http://web.media.mit.edu/ justine/publications.html

Ryokai, K., Vaucelle, C. \& Cassell, J. (2002b). Virtual Peers as Partners in Storytelling and Literacy Learning. Journal of Computer Assisted Learning.

UNESCO (2000). Informe Mundial sobre la Comunicación y la Información 1999-2000 (Academy for Educational Development). Washington DC.: Wadi D. Haddad \& Alexandra Draxler.

SEDICI (2000). Métrica de la Sociedad de la Información. Datos 1999-2000. SEDICl Asociación Española de Empresas de Tecnología de la Información. Disponible en http://www.campus-oei.org/salactsi/Metrica.PDF

Vallverdú, F., Sancho T., Mor E., Santanach, F. \& Abad, A. (1998). Agentes Inteligentes y Libros Digitales, Barcelona: Ediciones UOC. Disponible en http://cv.uoc.es/ grc0 000252 web/Articles/Pon Huelva.PDF

ww.campus-oei.org/salactsi/Metrica.PDF

Vallverdú, F., Sancho T., Mor E., Santanach, F. \& Abad, A. (1998). Agentes Inteligentes y Libros Digitales, Barcelona: Ediciones UOC. Disponible en http://cv.uoc.es/ grc0 000252 web/Articles/Pon Huelva.PDF 